\title{
ESWT for tendinopathy: technology and clinical implications
}

\author{
Henk van der Worp • Inge van den Akker-Scheek • \\ Hans van Schie $\cdot$ Johannes Zwerver
}

Received: 16 September 2011 / Accepted: 12 April 2012/Published online: 1 May 2012

(C) The Author(s) 2012. This article is published with open access at Springerlink.com

\begin{abstract}
Purpose The general consensus that tendinopathy, at least in the chronic stage, is mainly a degenerative condition and inflammation plays a minor role has led to a shift from treatments that target inflammation towards treatment options that promote regeneration. One of these treatments is extracorporeal shockwave therapy (ESWT), a physical therapy modality that uses pressure waves to treat tendinopathy. This review was undertaken to give an overview of the literature concerning this treatment, and special attention is given to the differences between focused and radial ESWT.

Methods A narrative description of wave characteristics, generation methods and in vitro effects of ESWT is given. The literature on ESWT as a treatment for one common tendinopathy, patellar tendinopathy, was systematically reviewed.

Results Waves that are generated for focused and radial ESWT have very different physical characteristics. It is unclear how these characteristics are related to clinical

Electronic supplementary material The online version of this article (doi:10.1007/s00167-012-2009-3) contains supplementary material, which is available to authorized users.

H. van der Worp $(\varangle)$. I. van den Akker-Scheek · J. Zwerver Center for Sports Medicine, University Medical Center Groningen, University of Groningen, P.O. Box 30.001, 9700 RB Groningen, The Netherlands

e-mail: h.van.der.worp@umcg.nl

H. van Schie

Department of Orthopaedics, Erasmus University Medical Center, Rotterdam, The Netherlands

H. van Schie

Department of Equine Sciences, Faculty of Veterinary Medicine, Utrecht University, Utrecht, The Netherlands
\end{abstract}

effectiveness. Studies into the biological effects of ESWT have mainly used focused shockwave therapy, showing a number of effects of shockwaves on biological tissue. The systematic review of studies into the clinical effects of ESWT for patellar tendinopathy showed conflicting evidence for its effectiveness.

Conclusion Physical characteristics of focused and radial waves differ substantially, but effect on clinical effectiveness is unclear. Whereas in vitro studies often show the effects of ESWT on tendon tissue, results of clinical studies are inconsistent. Based on the review of the literature, suggestions are given for the use of ESWT in clinical practice regarding timing and treatment parameters. Level of evidence IV.

Keywords Tendinopathy $\cdot$ Shockwave $\cdot$ Treatment

\section{Introduction}

Tendon injuries (tendinopathies) are common in the entire population, especially in relation to sports and occupation $[45,46]$. Tendinopathy has a complex pathophysiology. It consists of a short acute inflammatory stage but after some time, it gradually becomes a degenerative condition [1].

Because both conservative and surgical management of tendinopathy is not always successful, new treatment modalities have been developed. One of these modalities is extracorporeal shockwave therapy (ESWT). In 2002, Chung and Wiley [8] published a review about ESWT for treating tendinopathies. At that time, they concluded based on the literature that there was strong evidence for the effectiveness of ESWT for chronic tendinopathy and that further research was required to settle debates concerning 
applied energy, number of pulses and number of treatment sessions.

Over the last decade, next to increased knowledge about the pathogenesis of tendinopathy, there have been technical developments and an accumulation of studies examining the working mechanisms of ESWT and its effectiveness. One of the main technical developments is that nowadays two different kinds of ESWT are used for treating tendinopathy: focused ESWT (FSWT) and radial ESWT (RSWT). RSWT is relatively new and has made ESWT more affordable and more widely available. These new technologies are the rationale for this review. Most research has been done using FSWT, but research on RSWT is starting to be published. The aim of the present review is to give an up-to-date description of ESWT, with a special focus on differences between FSWT and RSWT, and review the literature about this treatment method. The overview consists of a description of wave characteristics, methods to generate shockwaves, and in vitro and clinical effects of ESWT, the latter by performing a systematic review with methodological quality assessment on the effects of ESWT for patellar tendinopathy, as an example of a common tendinopathy.

\section{Pressure waves}

Pressure waves (or sound waves) are oscillating mechanical waves that can travel through gas, liquids and solids. A shockwave is a special, non-linear type of pressure wave (Fig. 1), characterized by a short rise time. The total duration of a shockwave is around $10 \mu$ s [10, 42].

Both the positive and the negative phase of a shockwave have an effect on interfaces between tissues with different density (acoustic impedance). During the positive phase, shockwaves with high pressure may hit an interface, leading to reflections, or they may pass and gradually become absorbed. The negative (tensile) phase of the shockwave causes cavitation at the tissue interfaces. During cavitation, air bubbles are formed as a result of the negative pressure. These bubbles subsequently implode

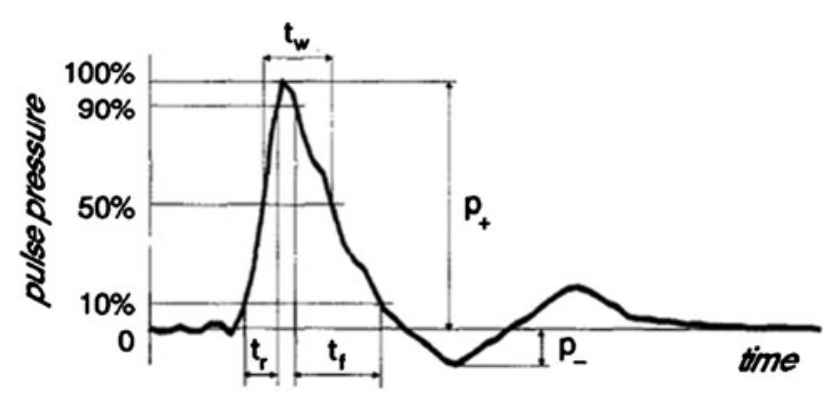

Fig. 1 Pressure-time profile of a shockwave (reprinted from [6]) with high speed, generating a second wave of shockwaves or micro-jets of fluid [10, 42].

\section{Types of ESWT}

There are two types of shockwave therapy: focused shockwave therapy (FSWT) and radial shockwave therapy (RSWT). This section will describe wave characteristics of both methods.

\section{FSWT}

FSWT is called focused because a pressure field is generated that converges in the adjustable focus at selected depth in the body tissues, where the maximal pressure is reached (Fig. 2a). There are three methods to generate focused shockwaves for FSWT: electrohydraulic (EH), electromagnetic (EM) and piezoelectric (PE) [42]. All three have in common that the waves are generated in water (inside the applicator). Focused shockwaves are generated in water because the acoustic impedance of water and biologic tissue is comparable. As a result of this, reflection is limited and waves are better transferred into the body.

A difference between these three methods is the moment at which the shockwave forms. EH generators produce
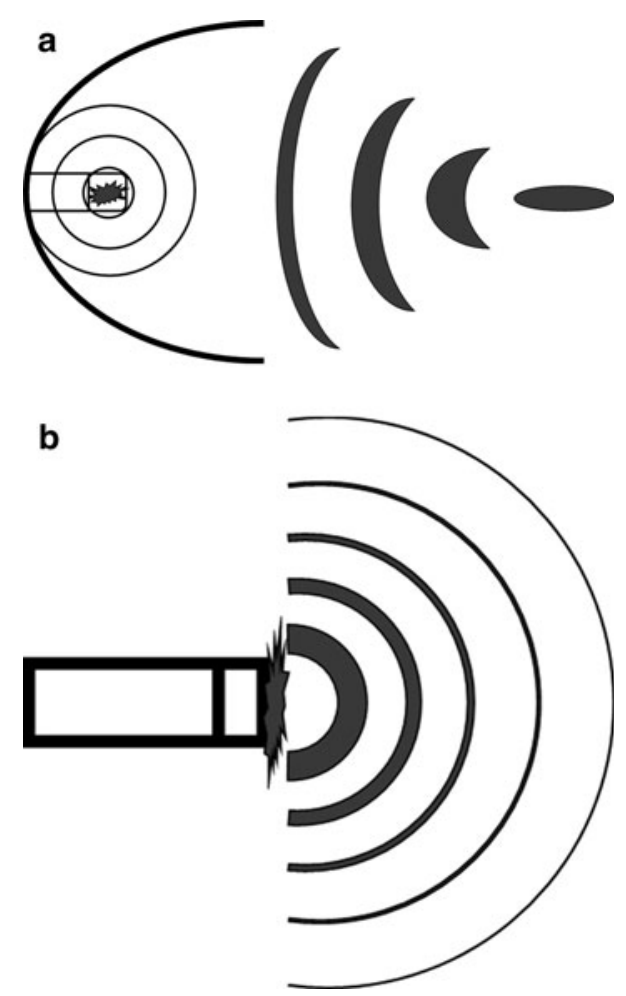

Fig. 2 a Pressure field of a focused shockwave device (EH-generated by means of spark gap). b Pressure field of a radial shockwave device 
focused shockwaves at origin, immediately after the spark gap, while EM and PE generators form shockwaves nanoseconds later by means of focusation of waves that are generated [12].

\section{RSWT}

The term radial refers to the diverging pressure field of RSWT devices, which reach a maximal pressure already at the source (Fig. 2b), not at a selected depth in the body. Radial shockwaves for RSWT are generated by accelerating a projectile, using compressed air, through a tube on the end of which an applicator is placed. The projectile hits the applicator and the applicator transmits the generated pressure wave into the body. In contrast to focused shockwave, radial pressure waves are not generated in water.

\section{FSWT versus RSWT}

There are two important differences in wave characteristics between focused shockwaves and radial shockwaves. First, radial shockwaves have a more superficial effect, compared to focused shockwaves, which reach a maximal energy in the focus that is located deeper into the body tissues (Fig. 2) [39]. It was shown that a RSWT device generates a pressure field extending to $40 \mathrm{~mm}$ in water, whereas the pressure field generated during FSWT may reach a distance that is about twice as high [39]. How these measures relate to biological tissue is not known. These measures are also dependent of the device that is used and the energy setting. In general, focused shockwaves will travel further and have more impact on deeper located tissues.

Second, research has shown that pressure waves generated by RSWT from a fundamental point of view cannot be called shockwaves because they lack the characteristic physical features of shockwaves (Fig. 3) such as a short rise time, a high peak pressure and non-linearity [11]. A

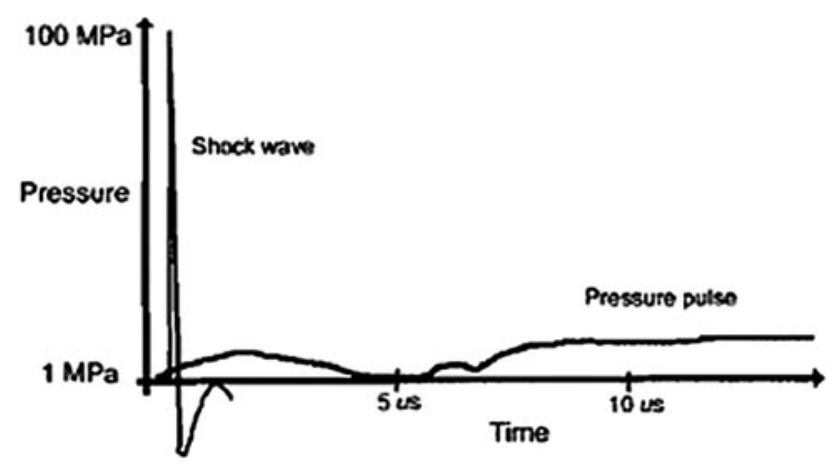

Fig. 3 Differences in pressure-time profile of a shockwave (generated with a focused shockwave device) and a pressure wave (generated with a radial shockwave device) (reprinted from [39]) reason for this is that the speed of sound in tissue is around $1,500 \mathrm{~m} / \mathrm{s}$, whereas the projectile during radial pressure wave generation can only reach a speed of around $20 \mathrm{~m} / \mathrm{s}$ [39]. This speed is not high enough to generate a real shockwave. Chitniss and Cleveland [7] found that the rise time (tr) of the generated wave was 25-40 ns for two focused devices $(\mathrm{EH})$, whereas it was $600 \mathrm{~ns}$ for a radial shockwave device. Although $25-40 \mathrm{~ns}$ is longer than the definition given above of a shockwave, the waves generated with the EH devices showed the features that are typical for a shockwave (Fig. 1), whereas the wave generated with the radial device lacked these characteristics. Based on these findings, it may be more correct to use the term radial pressure wave therapy instead of RSWT. Radial pressure wave devices also come with 'focused' applicators. However, Cleveland et al. [11] showed that these applicators do not generate real shockwaves either.

Because it is not clear which wave characteristics generate therapeutic effects, it is difficult to relate physical differences between focused shockwaves and radial pressure waves to clinical effectiveness $[9,36]$.

\section{Biological effects of ESWT-in vitro studies}

Until now, most fundamental research on ESWT for tendinopathy has been done with focused shockwaves. Fundamental research into the biological effects of ESWT has been concentrated on a number of non-exclusive theories about the working mechanisms of ESWT in tendinopathy. These theories can be roughly divided into pain relief, tissue regeneration and destruction of calcifications.

\section{Pain relief}

Pain relief with ESWT might work by means of hyperstimulation analgesia [40]. Overstimulation of the treated site would lead to a diminished transmission of signals to the brainstem [51]. Animal studies show that ESWT has an influence on pain transmission by acting on substance $\mathrm{P}$ [21, 37], calcitonin gene-related peptide (CGRP) expression in the dorsal root ganglion [52] and on neurovascular sprouting [20], Haake et al. however found no effect of ESWT on substance P and CGRP [18].

Tissue regeneration

A second theory is that ESWT stimulates tissue regeneration. Tissue regeneration by means of ESWT does fit within the framework of mechanotransduction, where mechanical load on the cytoskeleton leads to cell responses and increased protein synthesis [26]. Healthy human tenocytes responded to ESWT with cell growth and 
increased collagen synthesis [55], mainly type-I, and in affected human tenocytes, ESWT decreased the expression of matrix metalloproteases (MMPs) and interleukins (ILs) that are associated with tendinopathy [19]. Animal studies show that ESWT leads to an increase in collagen production and matrix turnover [3, 4, 23], increased vascularization in the bone-tendon junction [57] and increased tissue regeneration in wound healing and ischaemia $[25,31,41]$.

Destruction of calcifications

Although in vitro studies are lacking, it is thought that ESWT may also destroy calcifications in tendons. This effect is comparable with the way shockwaves are used in lithotripsy to destroy kidney stones. In vivo studies show the disintegration of calcifications in shoulder tendinopathy after ESWT [15, 44].

\section{Clinical effects of ESWT}

Although in vitro studies have demonstrated biological effects of ESWT, clinical effects of ESWT are less clear. In this section, we will focus on patellar tendinopathy, as an example of a common sports injury for which ESWT is increasingly used and which has the same underlying pathology as other common (insertional) tendinopathies [27]. A systematic search of the literature was performed to identify randomized controlled trials (RCTs) that studied the effectiveness of ESWT for patellar tendinopathy. The search was performed in the PubMed and Embase database. Four RCTs were found in this search $[43,53,56,58]$. The methodological quality of the four indentified studies was independently scored by two authors (Henk van der Worp and Inge van den AkkerScheek) using the PEDro checklist [35]. Characteristics as well as the PEDro score of the four included studies are shown in Table 1.

From this table, it appears that, although in vitro studies have demonstrated biological effects of ESWT, the clinical effects of ESWT for the treatment of patellar tendinopathy are less clear. Some studies found ESWT to be effective, whereas in others there was no or little improvement. Remarkably, the study that showed the largest improvement was the only one without a placebo intervention [56].

\section{Discussion}

The most important finding of this review was that there is conflicting evidence regarding the effectiveness of ESWT for patellar tendinopathy. This conflicting evidence may have several reasons. First, there is a lack of objective diagnostic criteria for patellar tendinopathy. Second, it may be that ESWT is only effective during certain stages of tendinopathy and not during other stages. A third reason may be that there are many instrumental settings-like choice of generator (EH, EM or PE), focal depth, number and intensity of pulses (energy flux density) - that can be varied and which may play a role in the effectiveness. A last reason is a methodological one.

These four topics will be described below. These topics are also of importance for research into the effectiveness of other tendinopathies where also conflicting results have been shown $[2,5,54]$.

\section{Diagnosis}

There is no gold standard for the diagnosis of tendinopathy. This diagnosis is obtained from a combination of history of symptoms and physical examination [14]. Imaging increases the likelihood of a correct diagnosis, but is not conclusive. The absence of a gold standard may result in non-uniform populations in clinical studies.

Stage of tendinopathy

Effectiveness of ESWT may depend on the stage of tendinopathy. A recent model of tendinopathy differentiates between a reactive tendinopathy/early tendon disrepair phase and a late tendon disrepair/degeneration phase [13]. ESWT seems most appropriate in the latter where the tendinopathy is degenerative and when conservative treatment has no effect $[13,45]$. This is also supported by recent studies that showed no effect of ESWT in the early stage of tendinopathy $[47,58]$. Until now, studies have not differentiated between subjects in the study based on these different stages; therefore, different studies may have used populations that are not comparable.

\section{Treatment parameters}

There are a number of instrumental settings that can be varied during ESWT (Table 2). The exact relationship between these settings and the effectiveness of the treatment are often unclear, although for some settings there is some indication as to how they may influence effectiveness.

Energy flux densities above $0.50 \mathrm{~mJ} / \mathrm{mm}^{2}$ should be avoided [38, 51]. Bosch et al. [3] showed in an animal study that EH-generated shockwaves already have a major impact on healthy tendon tissue at an intensity of 0.14 $\mathrm{mJ} / \mathrm{mm}^{2}$ [3].

Little is known about the optimal number of impulses in tendinopathy, one study showed that three treatments with 500 impulses were more effective than three treatments with 100 impulses in plantar fasciitis [30]. 


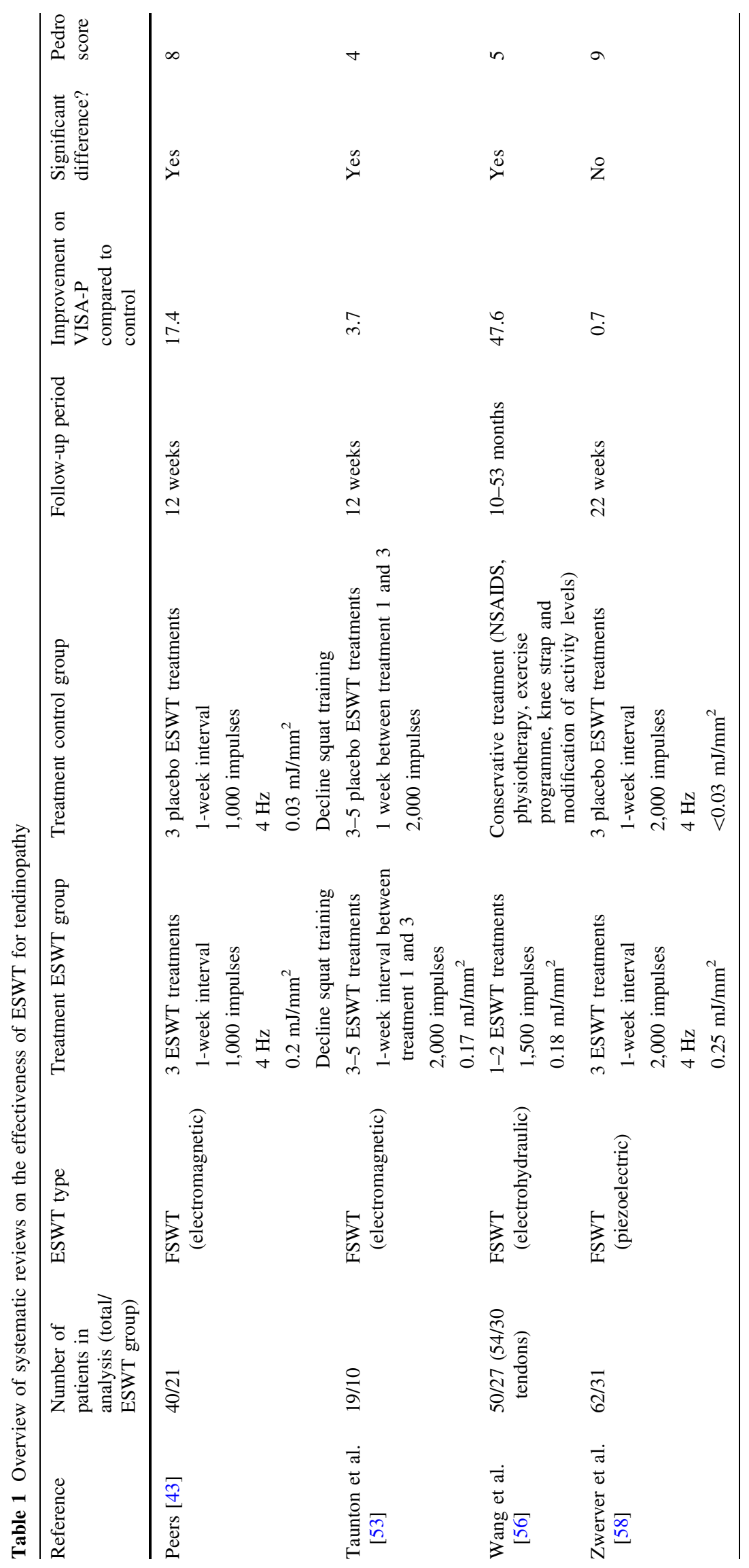


Table 2 Treatment parameters

\begin{tabular}{lc}
\hline Treatment parameters & Description \\
\hline Maximal positive pressure & $\begin{array}{c}\text { The maximal positive pressure } \\
\text { that is reached } \\
\text { A 3-D ellipsoid where the } \\
\text { pressure is above a } \\
\text { certain value } \\
\text { The amount of energy/surface } \\
\text { unit }\left(\mathrm{mJ} / \mathrm{mm}^{2}\right)\end{array}$ \\
$\begin{array}{l}\text { Energy flux density } \\
\text { Time interval between } \\
\text { treatments }\end{array}$ & $\begin{array}{c}\text { The number of shockwaves } \\
\text { that is applied/second }\end{array}$ \\
$\begin{array}{l}\text { Impulse frequency } \\
\text { How the to-be-treated } \\
\text { site is determined? }\end{array}$ \\
$\begin{array}{l}\text { Anaesthesia } \\
\text { Concurrent treatments/rest }\end{array}$
\end{tabular}

High frequencies do not seem advisable as cavitation bubbles may block the propagation of subsequent waves [10], and the maximum generated pressure seems to drop [12].

Localization of the site that needs treatment can be determined by means of palpation, ultrasound or radiographs. The relationship between these localization methods and pathology is not always clear though [22, 28, 33].

The use of anaesthesia during ESWT seems not advisable as three studies comparing ESWT with and without anaesthesia showed that treatment without anaesthesia is more effective [16, 32, 50].

Rest seems to be important in the first phase after ESWT treatment. Heavy physical activities are best avoided in this phase because the tendon can bear less load shortly after ESWT [3]. This is in line with a recent study that showed no effect of ESWT in actively competing athletes [58]. Although research is scarce, a combination of treatments may have a synergistic effect and lead to better results. Two studies found better results for a combination of ESWT and eccentric exercises than for eccentric exercises alone [43, 48]. Further research on these topics is required.

\section{Methodology}

To prevent that natural improvement, which may be possible in the early stages of tendinopathy, is mistaken for a treatment effect, it is important to include a placebo control group in ESWT effectiveness studies. Furthermore, studies should have a long enough follow-up time to discover treatment effects, since it is known that the metabolic turnover rate of tendon tissue is slow. These methodological issues may also explain some of the conflicting result found for the effectiveness of ESWT (Table 1).

\section{Clinical effectiveness of FSWT versus RSWT}

All four RCTs included in the systematic review on patellar tendinopathy used focused shockwave devices. This may be because radial shockwave devices have been introduced recently. Therefore, no conclusions can be drawn with regard to the effectiveness of RSWT for patellar tendinopathy. For plantar fasciitis, two RCTs have been published that looked at the effect of RSWT [17, 24]. Both studies found RSWT to be effective for this condition. No other placebo controlled studies on the effectiveness of RSWT for treating tendinopathy have been published. There is some evidence from non-placebo controlled studies that RSWT is effective for Achilles tendinopathy $[48,49]$.

Until now, only one study has directly compared the effectiveness of FSWT and RSWT [34], using both methods to treat plantar fasciitis, and a small difference in favour of FSWT was found. The authors do not hypothesize about what may be the cause of this difference though. Maybe FSWT was more effective because the plantar fascia is located deep in the body (compared to other tendons), so it is better reached with the waves generated by means of FSWT, which achieve their maximal energy within the focus. However, because RSWT also is shown to be effective for treating plantar fasciitis [17, 24], these waves, with a pressure field that reaches around $40 \mathrm{~mm}$ in water, probably also travel far enough in tissue to reach the affected area. It is therefore based on the present clinical literature not possible to recommend one of the two types of ESWT over the other.

\section{Conclusion}

Although evidence for the effectiveness of ESWT for treating tendinopathy is inconsistent, it is used widely in sports medicine. The present overview aimed at describing ESWT, in particular the two types that are used: FSWT and RSWT. Waves that are generated for FSWT and RSWT have very different physical characteristics. The relationship between these characteristics and clinical effectiveness is unclear. Studies into the biological effects of ESWT have mainly used FSWT, showing a number of effects of shockwaves on biological tissue. Clinical effects of ESWT for (patellar) tendinopathy are less clear. Reasons for this may be the non-uniform inclusion criteria related to the absence of a diagnostic gold standard, populations from different pathological stages, the large number of treatment parameters that can be varied and methodological issues.

It remains therefore questionable whether ESWT should be recommended at all. This is probably also the case for most other tendinopathies for which also conflicting 
findings regarding the effectiveness of ESWT have been reported. Further research is required to determine the value of ESWT for tendinopathy. This research should consist of a combination of in vitro and clinical studies. Studies with clear descriptions of study populations, diagnostic criteria and treatment parameters and concurrent rehabilitation programmes/tendon loading activities are necessary to advance research.

\section{Clinical implications}

This review provides some suggestions for the use of ESWT in clinical practice. When ESWT is used to treat tendinopathy, it seems best to apply it in a later stage [13], in combination with tendon load management [29], after other conservative options have been tried and before more radical options like surgery are considered. Based on the literature, low energy, a low frequency, no anaesthetics and exercise after an initial rest period can be recommended. At the moment, no recommendation can be given as to which of the two types of ESWT should be used.

The introduction of RSWT next to FSWT made ESWT more affordable and easier to administer. However, there is no agreement in the literature as to whether ESWT is effective for tendinopathy; hence, at the moment, there is no information available as to which of the two methods is preferable.

Open Access This article is distributed under the terms of the Creative Commons Attribution License which permits any use, distribution, and reproduction in any medium, provided the original author(s) and the source are credited.

\section{References}

1. Abate M, Silbernagel KG, Siljeholm C, Di Iorio A, De Amicis D, Salini V, Werner S, Paganelli R (2009) Pathogenesis of tendinopathies: inflammation or degeneration? Arthritis Res Ther 11: 235

2. Andres BM, Murrell GAC (2008) Treatment of tendinopathy: what works, what does not, and what is on the horizon? Clin Orthop Relat Res 466:1539-1554

3. Bosch G, de Mos M, van Binsbergen R, van Schie HTM, van de Lest CHA, van Weeren PR (2009) The effect of focused extracorporeal shock wave therapy on collagen matrix and gene expression in normal tendons and ligaments. Equine Vet $\mathbf{J} 41$ : 335-341

4. Bosch G, Lin YL, Van Schie HTM, Van de Lest CHA, Barneveld A, Van Weeren RR (2007) Effect of extracorporeal shock wave therapy on the biochemical composition and metabolic activity of tenocytes in normal tendinous structures in ponies. Equine Vet $\mathbf{J}$ 39:226-231

5. Buchbinder R, Green SE, Youd JM, Assendelft WJJ, Barnsley L, Smidt N (2006) Systematic review of the efficacy and safety of shock wave therapy for lateral elbow pain. J Rheumatol 33:1351-1363
6. Buizza A, Dell' Aquila T, Giribona P, Spagno C (1995) The performance of different pressure pulse generators for extracorporeal lithotripsy: a comparison based on commercial lithotripters for kidney stones. Ultrasound Med Biol 21(2):259-272

7. Chitnis PV, Cleveland R (2006) Acoustic and cavitation fields of shock wave therapy devices. AIP Conference Proceedings, 829: 440-444

8. Chung B, Wiley JP (2002) Extracorporeal shockwave therapy-a review. Sports Med 32:851-865

9. Cleveland RO, Chitnis PV, McClure SR (2008) Shock wave therapy: what really matters reply. Ultrasound Med Biol 34: 1869-1870

10. Cleveland RO (2007) The acoustics of shock wave lithotripsy. Ren Stone Dis 900:311-316

11. Cleveland RO, Chitnis PV, McClure SR (2007) Acoustic field of a ballistic shock wave therapy device. Ultrasound Med Biol 33:1327-1335

12. Coleman AJ, Saunders JE (1989) A survey of the acoustic output of commercial extracorporeal shock-wave lithotripters. Ultrasound Med Biol 15:213-227

13. Cook JL, Purdam CR (2009) Is tendon pathology a continuum? A pathology model to explain the clinical presentation of loadinduced tendinopathy. Br J Sports Med 43:409-416

14. Cook JL, Khan KM (2001) What is the most appropriate treatment for patellar tendinopathy? Br J Sports Med 35:291-294

15. Daecke W, Kusnierczak D, Loew M (2002) Long-term effects of extracorporeal shockwave therapy in chronic calcific tendinitis of the shoulder. J Should Elbow Surg 11:476-480

16. Furia JP (2006) High-energy extracorporeal shock wave therapy as a treatment for insertional Achilles tendinopathy. Am J Sports Med 34:733-740

17. Gerdesmeyer L, Frey C, Vester J, Maier M, Weil L Jr, Weil L Sr, Russlies M, Stienstra J, Scurran B, Fedder K, Diehl P, Lohrer H, Henne M, Gollwitzer H (2008) Radial extracorporeal shock wave therapy is safe and effective in the treatment of chronic recalcitrant plantar fasciitis results of a confirmatory randomized placebo-controlled multicenter study. Am J Sports Med 36: 2100-2109

18. Haake M, Thon A, Bette M (2002) No influence of low-energy extracorporeal shock wave therapy (ESWT) on spinal nociceptive systems. J Orthop Sci 7:97-101

19. Han SH, Lee JW, Guyton GP, Parks BG, Courneya J, Schon LC (2009) Effect of extracorporeal shock wave therapy on cultured tenocytes. Foot Ankle Int 30:93-98

20. Hausdorf J, Lemmens MAM, Heck KDW, Grolms N, Korr H, Kertschanska S, Steinbusch HWM, Schmitz C, Maier M (2008) Selective loss of unmyelinated nerve fibers after extracorporeal shockwave application to the musculoskeletal system. Neuroscience 155:138-144

21. Hausdorf J, Lemmens MAM, Kaplan S, Marangoz C, Milz S, Odaci E, Korr H, Schmitz C, Maier M (2008) Extracorporeal shockwave application to the distal femur of rabbits diminishes the number of neurons immunoreactive for substance $\mathrm{P}$ in dorsal root ganglia L5. Brain Res 1207:96-101

22. Hoksrud A, Ohberg L, Alfredson H, Bahr R (2008) Color Doppler ultrasound findings in patellar tendinopathy (jumper's knee). Am J Sports Med 36:1813-1820

23. Hsu RWW, Hsu WH, Tai CL, Lee KF (2004) Effect of shockwave therapy on patellar tendinopathy in a rabbit model. J Orthop Res 22:221-227

24. Ibrahim MI, Donatelli RA, Schmitz C, Hellman MA, Buxbaum F (2010) Chronic plantar fasciitis treated with two sessions of radial extracorporeal shock wave therapy. Foot Ankle Int 31:391-397

25. Ito $\mathrm{Y}$, Ito $\mathrm{K}$, Shiroto $\mathrm{T}$, Tsuburaya R, Yi GJ, Takeda M, Fukumoto Y, Yasuda S, Shimokawa H (2010) Cardiac shock wave therapy ameliorates left ventricular remodeling after myocardial 
ischemia-reperfusion injury in pigs in vivo. Coron Artery Dis 21:304-311

26. Khan KM, Scott A (2009) Mechanotherapy: how physical therapists' prescription of exercise promotes tissue repair. Br J Sports Med 43:247-252

27. Khan KM, Cook JL, Bonar F, Harcourt P, Astrom M (1999) Histopathology of common tendinopathies - update and implications for clinical management. Sports Med 27:393-408

28. Khan KM, Visentini PJ, Kiss ZS, Desmond PM, Coleman BD, Cook JL, Tress BM, Wark JD, Forster BB, Victorian Institute of Sport Tendon Study Group (1999) Correlation of ultrasound and magnetic resonance imaging with clinical outcome after patellar tenotomy: prospective and retrospective studies. Clin J Sports Med 9:129-137

29. Kountouris A, Cook J (2007) Rehabilitation of Achilles and patellar tendinopathies. Best Pract Res Clin Rheumatol 21: 295-316

30. Krischek O, Rompe JD, Herbsthofer B, Nafe B (1998) Symptomatic low-energetic shock-wave therapy in plantar fasciitis with heel spur. Z Orthop Ihre Grenzgeb 136:169-174

31. Kuo YR, Wang CT, Wang FS, Chiang YC, Wang CJ (2009) Extracorporeal shock-wave therapy enhanced wound healing via increasing topical blood perfusion and tissue regeneration in a rat model of STZ-induced diabetes. Wound Repair Regen 17:522-530

32. Labek G, Auersperg V, Ziernhold M, Poulios N, Bohler N (2005) Influence of local anesthesia and energy level on the clinical outcome of extracorporeal shock wave-treatment of chronic plantar fasciitis - a prospective randomized clinical trial. Z Orthop Ihre 143:240-246

33. Lian O, Holen KJ, Engebretsen L, Bahr R (1996) Relationship between symptoms of jumper's knee and the ultrasound characteristics of the patellar tendon among high level male volleyball players. Scand J Med Sci Sports 6:291-296

34. Lohrer H, Nauck T, Dorn-Lange NV, Schoell J, Vester JC (2010) Comparison of radial versus focused extracorporeal shock waves in plantar fasciitis using functional measures. Foot Ankle Int $31: 1-9$

35. Maher CG, Sherrington C, Herbert RD, Moseley AM, Elkins M (2003) Reliability of the PEDro scale for rating quality of randomized controlled trials. Phys Ther 83:713-721

36. Maier M, Schmitz C (2008) Shock wave therapy: what really matters? Ultrasound Med Biol 34:1868-1869

37. Maier M, Averbeck B, Milz S, Refior HJ, Schmitz C (2003) Substance $\mathrm{P}$ and prostaglandin E-2 release after shock wave application to the rabbit femur. Clin Orthop 406(1):237-245

38. Maier M, Tischer T, Milz S, Weiler C, Nerlich A, Pellengahr C, Schmitz C, Refior HJ (2002) Dose-related effects of extracorporeal shock waves on rabbit quadriceps tendon integrity. Arch Orthop Trauma Surg 122:436-441

39. McClure S, Dorfmuller C (2003) Extracorporeal shock wave therapy: theory and equipment. Clin Tech Equine Pract 2:348357

40. Melzack R (1979) Sensory modulation of pain. Int Rehabil Med $1: 111-115$

41. Mittermayr R, Hartinger J, Antonic V, Meinl A, Pfeifer S, Stojadinovic A, Schaden W, Redl H (2011) Extracorporeal shock wave therapy (ESWT) minimizes ischemic tissue necrosis irrespective of application time and promotes tissue revascularization by stimulating angiogenesis. Ann Surg 253:1024-1032
42. Ogden JA, Toth-Kischkat A, Schultheiss R (2001) Principles of shock wave therapy. Clin Orthop Rel Res 387:8-17

43. Peers KHE (2003) Extracorporeal shock wave therapy in chronic achilles and patellar tendinopathy. Dissertation or thesis, KU Leuven (ISBN: 9058673049)

44. Peters J, Luboldt W, Schwarz W, Jacobi V, Herzog C, Vogl TJ (2004) Extracorporeal shock wave therapy in calcific tendinitis of the shoulder. Skeletal Radiol 33:712-718

45. Rees JD, Maffulli N, Cook J (2009) Management of tendinopathy. Am J Sports Med 37:1855-1867

46. Rees JD, Wilson AM, Wolman RL (2006) Current concepts in the management of tendon disorders. Rheumatology 45:508-521

47. Rompe JD, Cacchio A, Haist J, Reiners V, Furia JP, Schmitz C, Weil L, Maffulli N (2010) Plantar fascia-specific stretching versus radial shock-wave therapy as initial treatment of plantar fasciopathy. J Bone Jt Surg Am 92:2514-2522

48. Rompe JD, Furia J, Maffulli N (2009) Eccentric loading versus eccentric loading plus shock-wave treatment for midportion Achilles tendinopathy: a randomized controlled trial. Am J Sports Med 37:463-470

49. Rompe JD, Furia J, Maffulli N (2008) Eccentric loading compared with shock wave treatment for chronic insertional Achilles tendinopathy. J Bone Jt Surg Am 90:52-61

50. Rompe JD, Meurer A, Nafe B, Hofmann A, Gerdesmeyer L (2005) Repetitive low-energy shock wave application without local anesthesia is more efficient than repetitive low-energy shock wave application with local anesthesia in the treatment of chronic plantar fasciitis. J Orthop Res 23:931-941

51. Rompe JD, Kirkpatrick CJ, Kullmer K, Schwitalle M, Krischek O (1998) Dose-related effects of shock waves on rabbit tendo Achillis-A sonographic and histological study. J Bone Jt Surg $\mathrm{Br} 80: 546-552$

52. Takahashi N, Wada Y, Ohtori S, Saisu T, Moriya H (2003) Application of shock waves to rat skin decreases calcitonin generelated peptide immunoreactivity in dorsal root ganglion neurons. Auton Neurosci 107:81-84

53. Taunton KM, Taunton JE, Khan KM (2003) Treatment of patellar tendinopathy with extracorporeal shock wave therapy. BC Med J 45:500-507

54. Thomson CE, Crawford F, Murray GD (2005) The effectiveness of extra corporeal shock wave therapy for plantar heel pain: a systematic review and meta-analysis. BMC Musculoskelet Disord 6:19

55. Vetrano M, d'Alessandro F, Torrisi MR, Ferretti A, Vulpiani MC, Visco V (2011) Extracorporeal shock wave therapy promotes cell proliferation and collagen synthesis of primary cultured human tenocytes. Knee Surg Sports Traumatol Arthrosc 19:2159-2168

56. Wang CJ, Ko JY, Chan YS, Weng LH, Hsu SL (2007) Extracorporeal shockwave for chronic patellar tendinopathy. Am J Sports Med 35:972-978

57. Wang CJ, Wang FS, Yang KD, Weng LH, Hsu CC, Huang CS, Yang LC (2003) Shock wave therapy induces neovascularization at the tendon-bone junction - a study in rabbits. J Orthop Res 21:984-989

58. Zwerver J, Hartgens F, Verhagen E, van der Worp H, van den Akker-Scheek I, Diercks RL (2011) No effect of extracorporeal shockwave therapy on patellar tendinopathy in jumping athletes during the competitive season: a randomized clinical trial. Am J Sports Med 39:1191-1199 